\title{
Revista Colombiana de

\section{Síndrome de apnea/hipopnea obstructiva del sueño y su asociación con las enfermedades cardiovasculares}

\author{
Nimrod S. Barashi ${ }^{a, *}$, Rafael E. Ruiz ${ }^{a}$, Lisseth Marín ${ }^{\mathrm{b}}$, Paula Ruiz ${ }^{\mathrm{b}}$, Sandra Amado ${ }^{\mathrm{a}, \mathrm{b}}$, \\ Álvaro J. Ruiz ${ }^{a, b, c}$ y Patricia Hidalgo ${ }^{a, d}$
}

a Facultad de Medicina, Pontificia Universidad Javeriana, Bogotá, D.C., Colombia

b Departamento de Medicina Interna, Hospital Universitario San Ignacio, Bogotá, D.C., Colombia

c Departamento de Epidemiología Clínica, Hospital Universitario San Ignacio, Bogotá, D.C., Colombia

' Unidad de Neumología, Departamento de Medicina interna, Hospital Universitario San Ignacio, Bogotá, D.C., Colombia

Recibido el 3 de abril de 2014; aceptado el 4 de agosto de 2014

Disponible en Internet el 21 de abril de 2015

\section{PALABRAS CLAVE \\ Sueño; \\ Factores de riesgo \\ cardiovascular; \\ Enfermedades \\ cardiovasculares; \\ Síndrome metabólico}

\begin{abstract}
Resumen
Introducción: El síndrome de apnea/hipopnea obstructiva del sueño, es una entidad que ha cobrado importancia en los últimos años, con una prevalencia estimada en adultos de edad media cercana al 4 y al $2 \%$ en hombres y mujeres, respectivamente, y que por su frecuencia constituye un problema de salud pública.

Objetivo: Exponer, tras un análisis exhaustivo de la literatura disponible, la asociación entre el síndrome de apnea/hipopnea obstructiva del sueño y las enfermedades cardiovasculares.

Método: Se hizo una revisión narrativa a partir de la literatura encontrada en las bases de datos más reconocidas. Se incluyeron 59 estudios publicados en los últimos treinta años y se excluyeron reportes y series de casos.

Conclusiones: El síndrome de apnea/hipopnea obstructiva del sueño se reconoce hoy en día como un problema de salud pública mundial. En Latinoamérica, más específicamente en Colombia, se requieren estudios prospectivos de cohorte que sirvan de pauta para la población del continente e indiquen posibles diferencias respecto a la comunidad internacional en cuanto a su tratamiento y diagnóstico oportunos, así como acerca del impacto de estos en lo concerniente a los desenlaces cardiovasculares de los pacientes.

(c) 2015 Sociedad Colombiana de Cardiología y Cirugía Cardiovascular. Publicado por Elsevier España, S.L.U. Este es un artículo Open Access bajo la licencia CC BY-NC-ND (http:// creativecommons.org/licenses/by-nc-nd/4.0/).
\end{abstract}

\footnotetext{
* Autor para correspondencia.

Correo electrónico: nimrodbarashi@gmail.com (N.S. Barashi).
} 


\section{KEYWORDS}

Sleep;

Cardiovascular risk

factors;

Cardiovascular

diseases;

Metabolic syndrome

\section{Obstructive Sleep Apnea Hypopnea Syndrome and its association} with cardiovascular disease

\begin{abstract}
Introduction: The Obstructive Sleep Apnea Hypopnea Syndrome has gained importance has gained importance in recent years, with an estimated prevalence in population of middle-aged adults around 4 and $2 \%$ in men and women respectively, and that given its frequency constitutes a public health problem.

Objective: To show, after a thorough analysis of the available literature, the association between the Obstructive Sleep Apnea Hypopnea Syndrome and cardiovascular diseases.

Method: A narrative review was made from the literature found at the most recognized databases. Fifty nine studies published in the last thirty years were included and reports and case series were excluded.

Conclusions: The Obstructive Sleep Apnea Hypopnea Syndrome is recognized today as a global public health problem. Latin America, specifically Colombia, requires prospective cohort studies that serve as a guideline for the continent's population and that could indicate possible differences compared to the international community regarding early diagnosis and treatment, and its impact in cardiovascular outcomes of these patients.

(c) 2015 Sociedad Colombiana de Cardiología y Cirugía Cardiovascular. Published by Elsevier España, S.L.U. This is an open access article under the CC BY-NC-ND license (http:// creativecommons.org/licenses/by-nc-nd/4.0/).
\end{abstract}

\section{Introducción}

El síndrome de apnea/hipopnea obstructiva del sueño (SAHOS) es una entidad frecuente, con una prevalencia en adultos de edad media cercana al 4 y $2 \%$ en hombres y mujeres, respectivamente. En personas asintomáticas puede ser tan alta como $24 \%$ en hombres y $9 \%$ en mujeres, y tiende a incrementar con la edad, según se reporta en la cohorte de Wisconsin ${ }^{1}$.

Su diagnóstico se realiza cuando, a partir de una sospecha clínica derivada de síntomas como somnolencia diurna excesiva, sueño no reparador, ronquidos y otros, se demuestra, a través de un polisomnograma, un índice de apnea/hipopnea (IAH) $\geq 5$ por hora. Este índice permite clasificar el SAHOS de acuerdo a su severidad, en leve (5-15 eventos/hora), moderado (16-30 eventos/hora) o severo (>30 eventos/hora), clasificación que ha mostrado utilidad tanto para la formulación de estrategias terapéuticas en estos pacientes, como para establecer su pronóstico.

En la actualidad está claro que el SAHOS es una entidad cuyas repercusiones no se limitan al aparato respiratorio, sino a múltiples sistemas, entre ellos el cardiovascular. Esta asociación se ha demostrado en varios trabajos llevados a cabo en los últimos años, entre los que sobresale el «Sleep Heart Health Study» (SHHS), un estudio prospectivo de cohorte que incluyó aproximadamente 6.600 pacientes adultos y cuyo objetivo fue evaluar si el SAHOS y otros trastornos del sueño constituyen factores de riesgo independientes para el desarrollo de enfermedades cardio y cerebrovasculares.

Como consecuencia de los resultados positivos de este estudio, existe un interés creciente a nivel mundial por demostrar si este síndrome es un factor de riesgo independiente para desarrollar enfermedades como hipertensión arterial sistémica, enfermedad coronaria, falla cardiaca, accidentes cerebrovasculares y síndrome metabólico ${ }^{1,2}$

De igual forma, se ha descrito que la morbilidad y mortalidad de pacientes con SAHOS son altas e independientes de diversos factores de confusión como obesidad, tabaquismo y alcoholismo, entre otros. Un estudio demostró que los pacientes con SAHOS tienen aproximadamente cinco veces más riesgo de desarrollar enfermedad cardiovascular en un seguimiento de siete años, independiente de la edad, el índice de masa corporal y la presión arterial ${ }^{3}$. En 2011, a partir de la cohorte de uno de los estudios Multi Ethnic Study of Atherosclerosis, se demostró que los pacientes con SAHOS tienen mayor incidencia de eventos cardiovasculares en comparación con los roncadores habituales (IC $95 \% 1,91(1,20-3,04), p=0,007)^{4}$. También se ha demostrado que el tratamiento con presión positiva continua en las vías respiratorias (CPAP) retrasa dicha morbimortalidad y mejora varios componentes que acompañan el síndrome.

\section{Método}

Se hizo una revisión narrativa a partir de la literatura encontrada en las bases de datos MedLine, Embase, LILACS y SciELO, utilizando los términos MeSH: «Sleep Apnea, Obstructive», «Cardiovascular Diseases», «Myocardial Infarction», «Heart Failure», «Sleep Apnea Syndrome», «Hypertension», «Cerebrovascular disorders»y «Metabolic Syndrome X».

En esta revisión se incluyeron 59 estudios publicados en los últimos treinta años, y se excluyeron reportes y series de casos. 


\section{Desarrollo}

\section{Fisiopatología del síndrome de apnea/hipopnea del sueño y el sistema cardiopulmonar}

A partir de la fragmentación del sueño y de la hipoxia intermitente que se evidencian en los pacientes con SAHOS, se desencadenan múltiples mecanismos fisiopatológicos responsables de la disfunción cardiometabólica. Durante los ciclos de hipoxia y reoxigenación se liberan especies reactivas de oxígeno y se desencadenan reacciones inflamatorias. Así mismo, se encuentran niveles elevados de sustancias como endotelina, factor inhibidor del plasminógeno 1, VCAM-1 e ICAM-1 y citoquinas inflamatorias (FNT $\alpha$, IL-1, IL-6 e IL-8), y hay disminución en los niveles de óxido nítrico en sangre.

Como consecuencia de estos ciclos de hipoxia y reoxigenación, hay una sobreproducción de peróxido de hidrógeno, anión superóxido y peroxinitrito, moléculas que en condiciones normales funcionan como segundos mensajeros en la señalización intracelular, pero que en exceso generan daño celular y tisular. Además de ello, se produce saturación y disminución de los mecanismos antioxidantes fisiológicos del cuerpo, entre los que destaca la acción de la albúmina. Este desequilibrio entre la producción de radicales libres y sistemas antioxidantes se manifiesta en peroxidación lipídica, oxidación de moléculas de lipoproteína de baja densidad (LDL), disfunción de células beta pancreáticas, lesión mitocrondrial y disminución de la biodisponibilidad del óxido nítrico para la formación de nuevos radicales libres ${ }^{5,6}$. Todo esto trae como consecuencia disfunción endotelial y predispone a la formación de placas ateroscleróticas, así como a la aparición de resistencia a la insulina y de diabetes mellitus, factores de riesgo importantes para el desarrollo de enfermedades cardiovasculares ${ }^{1,7-10}$.

Por otra parte, a raíz de los episodios de hipoxemia aguda hay retroalimentación por parte de los quimiorreceptores (en los cuerpos aórtico y carotídeo), lo que aumenta el tono simpático periférico, así como el tono adrenal y renal. Como consecuencia, no solo ocurre vasoconstricción y por ende hipertensión arterial secundaria, sino que se involucran otras vías como el sistema renina-angiotensinaaldosterona, la vía de la insulina y la adiponectina. Debido a los cambios de presión arterial, se genera un reajuste en los barorreceptores que hace que las cifras de presión altas se perciban como normales y se produzca menor disminución de la tensión arterial durante el sueño (non-dippers), fenómeno que aumenta el riesgo cardiovascular y en algunos casos, el de muerte súbita nocturna ${ }^{8,9,11}$.

Cada episodio de apnea genera un esfuerzo respiratorio significativo para el paciente, que conduce al aumento de la presión negativa intratorácica y a la alteración de los volúmenes circulatorios. El aumento de la presión transmural sobre el corazón y los grandes vasos, así como la disfunción endotelial y elevación de la resistencia vascular periférica por incremento del tono simpático, conlleva un aumento en el volumen de fin de sístole y, por tanto, del trabajo ventricular. Así mismo, el aumento del retorno venoso y de la resistencia de la arteria pulmonar dada por los episodios de hipoxia, lleva a dilatación de las cavidades derechas y a desviación del septum interventricular hacia el ventrículo izquierdo, disminuyendo así su volumen de llenado. Estos procesos causan ciertas modificaciones del tejido cardíaco, como remodelación y fibrosis miocárdica, que ocurren múltiples veces durante el sueño de estos pacientes y predisponen al desarrollo de consecuencias crónicas como disfunción ventricular, hipertrofia y dilatación de las cavidades cardíacas ${ }^{6,8,9,11,12}$.

En la actualidad no se conoce con exactitud la respuesta molecular a la hipoxemia y a la reoxigenación. Sin embargo, en modelos in vitro, cuando se comparan los ciclos con hipoxemia sostenida, se plantea un probable estrés celular por disfunción mitocondrial, acompañado de respuesta inflamatoria. Se cree que esta última está mediada por el factor de transcripción nuclear $\kappa \beta$, con efectos consecuentes en la expresión de factores que promueven la aterogénesis (como el FNT $\alpha$ ) y que causan disfunción endotelial y las consecuencias cardiovasculares que de ella se derivan. En cambio, en la hipoxia sostenida hay una respuesta adaptativa que va dirigida a mejorar la perfusión, con la liberación del factor inducible por hipoxia y el aumento en la expresión de genes que codifican proteínas como eritropoyetina, sintasa de óxido nítrico y factor de crecimiento endotelial vascular ${ }^{1}$.

Por otra parte, si bien es cierto que la obesidad no ha demostrado ser una causa independiente de SAHOS y que no todos los pacientes con este son obesos, esta condición se asocia con procesos inflamatorios que incluyen aumento de los niveles de PCR, de proteínas unidas a los ácidos grasos del adipocito y de ghrelina, además de alteraciones en la secreción de cortisol y leptina, que a su vez se relacionan con ganancia de peso y depresión respiratoria en los obesos. Así mismo, se han identificado niveles disminuidos de sustancias antiinflamatorias como IL-10 y adiponectina, esta última asociada con SAHOS severo, independiente de la obesidad $^{7}$.

\section{Hipertensión arterial sistémica}

La hipertensión arterial es tal vez la entidad que más se relaciona con SAHOS como un factor independiente, de ahí que en 2003 el VII Joint National Committee on Prevention, Detection, Evaluation, and Treatment of High Blood Pressure (JNC 7) decidiera incluir este síndrome como una causa de hipertensión arterial secundaria ${ }^{13}$. En la cohorte de sueño de Wisconsin se demostró relación estadísticamente significativa entre la severidad del SAHOS (dada por el IAH) y el desarrollo de hipertensión arterial sistémica, puesto que aquellos con IAH mayor a 15 eventos/hora tuvieron más riesgo $(O R=2,89, I C 95 \%=1,46-5,64)$ aún después de corregir variables de confusión como obesidad, consumo de medicamentos antihipertensivos, entre otras ${ }^{14}$. Sin embargo, Hass et al. demostraron que dicha asociación se pierde en pacientes mayores de 60 años ${ }^{15}$.

Numerosos estudios hasta la fecha han reportado una relación significativa entre el SAHOS y la hipertensión arterial refractaria, definida como la persistencia de cifras tensionales fuera de metas a pesar del manejo a dosis plenas con tres antihipertensivos de distintas clases, entre ellos un diurético. En 2005, Ryan et al. reportaron que aproximadamente el $83 \%$ de los pacientes con hipertensión arterial refractaria presentaron $\mathrm{SAHOS}^{1}$, mientras que en 2007 Gonçalves et al. obtuvieron cifras de aproximadamente 
$71 \%^{16}$. Por otra parte, se ha demostrado que la terapia con CPAP disminuye la presión arterial en pacientes con SAHOS, en especial con las formas moderada o severa ${ }^{17}$.

\section{Enfermedad cardíaca isquémica y arterioesclerosis}

Respecto a la relación entre SAHOS y enfermedad cardíaca isquémica, la información publicada a la fecha muestra resultados controversiales. Algunos estudios han descrito que la prevalencia de SAHOS en pacientes con antecedente de infarto agudo de miocardio es de 30 a $40 \%{ }^{18,19}$ y que este síndrome aumenta el riesgo de requerir procedimientos de revascularización coronaria o muerte por eventos cardiovasculares, independiente de otros factores de riesgo como hipertensión arterial ${ }^{20}$. No obstante, en 2012 se publicó una revisión sistemática y metaanálisis en la que, tras evaluar seis estudios con una muestra de 8.758 pacientes, no se encontró evidencia estadísticamente significativa que avalara la asociación entre enfermedad cardíaca isquémica y SAHOS $^{21}$. Cabe mencionar que si bien la asociación entre SAHOS y enfermedad cardíaca isquémica es incierta, se ha demostrado que el tratamiento con CPAP reduce el riesgo a largo plazo de muerte por enfermedad cardiovascular ${ }^{22}$.

Dentro del espectro de las consecuencias cardiovasculares que se evidencian en los pacientes con SAHOS, existe una asociación fuerte con el desarrollo temprano de arterioesclerosis. Como se explicó previamente, los ciclos de oxigenación y reoxigenación, que llevan a disfunción endotelial, son el mecanismo responsable del desarrollo de la misma. Igualmente, se ha propuesto que la vibración asociada con el ronquido puede ser causa directa de arterioesclerosis carotídea y que la hipertensión arterial, la resistencia a la insulina, la dislipidemia y otros procesos inflamatorios que se asocian con SAHOS, constituyen causas indirectas ${ }^{23}$. El vínculo entre SAHOS y esta enfermedad se ha demostrado a través de la medición de marcadores de remodelación arterial, rigidez arterial, grosor de las capas íntima y media en la carótida y velocidad de la onda de pulso ${ }^{9,24}$. Todo esto sugiere que el SAHOS debería considerarse como un factor de riesgo para enfermedad coronaria y cerebrovascular ${ }^{25}$.

En 2007 se llevó a cabo un estudio en pacientes con SAHOS severo sin otras comorbilidades cardíacas, en el que, tras un seguimiento de cuatro meses con CPAP, se demostró mejoría en los marcadores tempranos de arterioesclerosis, siendo un beneficio a corto plazo ${ }^{26}$. En cuanto al efecto del tratamiento con CPAP en las lesiones crónicas de la arterioesclerosis, no se sabe con claridad la magnitud del beneficio, sin embargo en 2009 Bayram et al. mostraron que la disfunción endotelial mediada por el flujo sanguíneo es reversible después de seis meses de tratamiento con CPAP ${ }^{27}$.

\section{Accidente cerebrovascular}

Incluso antes del SHHS varios investigadores sugirieron la asociación entre apnea del sueño y enfermedad cerebrovascular. En 1989, Palomäki et al. reportaron un OR de 8,0 para accidente cerebrovascular en sujetos con historia de SAHOS, aún después de corregir variables de confusión como hipertensión arterial, obesidad, consumo de bebidas alcohólicas y enfermedad coronaria ${ }^{28}$. Sin embargo este, así como varios estudios previos al SHHS, tiene validez limitada debido a que no se corroboró la presencia de SAHOS mediante polisomnograma ${ }^{29}$.

En la actualidad es claro que la apnea del sueño aumenta el riesgo de accidente cerebrovascular, eventos cardiovasculares fatales y no fatales, y muerte $21,30-32$. En uno de estos estudios, publicado en 2005 por Yaggi et al., se reportó que el riesgo de los pacientes con SAHOS para desarrollar ataque isquémico transitorio, accidente cerebrovascular y muerte es dos veces mayor que el de la población sin el síndrome ${ }^{32}$. Existe evidencia que valida que el tratamiento con CPAP disminuye el riesgo de enfermedad cerebrovascular fatal, por lo que podría ser una herramienta de prevención primaria ${ }^{22}$. Por el contrario, la efectividad del uso del CPAP para prevención terciaria todavía no se ha determinado ya que en los pacientes con SAHOS que presentaron un evento cerebrovascular, el IAH puede verse alterado por las consecuencias del accidente cerebrovascular sobre el control respiratorio ${ }^{14}$.

\section{Falla cardíaca}

La prevalencia de trastornos relacionados con el sueño en pacientes con falla cardíaca es alta. De acuerdo con resultados publicados en los últimos años, se estima que aproximadamente un 41 a $75 \%$ de los pacientes con falla cardíaca estable y tratada, presentarán trastornos del sueño, ya sea de origen obstructivo (31\%) o central (30\%) $)^{33,34}$. En 2001 , mediante un estudio transversal con el SHHS, se reportó la asociación del SAHOS con falla cardíaca con un OR de 2,38 ${ }^{35}$. La relación entre disfunción diastólica y SAHOS es clara y puede explicarse teniendo en cuenta que la principal causa de disfunción diastólica es la hipertensión arterial, fenómeno común en los pacientes con falla cardíaca y SAHOS. Sin embargo, la hipertensión arterial por sí sola no es suficiente para ser la responsable de la falla cardíaca, auque sí constituye un factor de riesgo establecido importante ${ }^{36}$.

Con base en los mecanismos fisiopatológicos que hacen referencia a alteraciones en los volúmenes circulatorios y en las cavidades cardíacas, es posible explicar cómo el SAHOS, en especial en pacientes con IAH mayor o igual a 15, es capaz de generar efectos deletéreos sobre la contractilidad ventricular derecha e izquierda ${ }^{37,38}$. Por otra parte, se ha demostrado que el tratamiento con CPAP genera beneficio en pacientes con falla cardíaca ya que disminuye la actividad simpática, la presión arterial e incluso la frecuencia cardíaca $^{39,40}$. En 2008 un grupo de investigadores en Madrid reportó que el tratamiento con CPAP mejora la fracción de eyección del ventrículo izquierdo, la variabilidad de la frecuencia cardíaca y la presión transmural ${ }^{41}$.

\section{Arritmias cardíacas}

La relación entre trastornos del sueño y arritmias cardíacas fue sugerida por primera vez en 1983 por Guilleminault et al., quienes observaron mayor prevalencia de episodios de fibrilación auricular, paro sinusal y bloqueos atrioventriculares en los pacientes con SAHOS ${ }^{42}$. Entre las investigaciones realizadas posteriormente, se destaca una cohorte del SHHS, en la que se observó que los pacientes con SAHOS tenían 
un riesgo relativo indirecto de 4,0 de sufrir fibrilación auricular ${ }^{43}$.

Los mecanismos fisiopatológicos propios del SAHOS, descritos anteriormente, conllevan alteraciones funcionales y estructurales que favorecen la aparición de trastornos de la conducción y del ritmo cardíaco en estos pacientes, especialmente de taquiarritmias como fibrilación auricular. Los episodios de hipoxia y el aumento del tono simpático favorecen la aparición de disfunción ventricular y posteriormente de remodelación miocárdica y dilatación auricular, que sirven como sustrato anatómico para la aparición de arritmias cardíacas. Así mismo, los cambios inflamatorios, la hipoxemia y las respuestas neurovegetativas pueden llevar a alteraciones en los tiempos de conducción o del período refractario auricular, entre otros ${ }^{44,45}$.

Por otra parte, se ha demostrado que el tratamiento con CPAP disminuye la recurrencia de arritmias cardíacas en pacientes con SAHOS. El estudio de Kanagala et al. demostró que los pacientes con SAHOS y fibrilación auricular que recibieron CPAP después de cardioversión eléctrica, tuvieron $82 \%$ menos recurrencias que aquellos que no ${ }^{46}$. El CPAP también ha mostrado reducir la recurrencia de fibrilación auricular en pacientes con SAHOS que fueron sometidos a ablación de venas pulmonares; así mismo disminuye la bradicardia sinusal y las pausas sinusales ${ }^{47,48}$.

\section{Síndrome metabólico y obesidad}

Al hablar de SAHOS y riesgo cardiovascular es preciso mencionar su asociación con el síndrome metabólico, pues es tan fuerte que se ha considerado incluirla como un componente más del síndrome metabólico, al que se le ha llamado «síndrome $Z$ ». Pese a que la obesidad es una condición en ocasiones compartida por el SAHOS y el síndrome metabólico, se ha encontrado una relación independiente de la apnea obstructiva del sueño con el desarrollo de resistencia a la insulina, dislipidemia e hipertensión arterial ${ }^{7,8}$.

Se ha demostrado una relación directa del SAHOS con varios de los componentes del síndrome metabólico. En cuanto a la dislipidemia, la evidencia se remonta al SHHS, que mostró relación inversa entre los niveles de HDL y el IAH, así como relación directa con los niveles de triglicéridos en hombres y mujeres menores de 65 años, independiente del índice de masa corporal ${ }^{7,49}$. En cuanto al desarrollo de resistencia a la insulina, intolerancia a los hidratos de carbono y diabetes mellitus tipo 2, varios estudios muestran resultados que favorecen su asociación ${ }^{50-52}$. En 2005 se llevó a cabo un estudio en el cual se restringió a los participantes el sueño nocturno a cuatro horas durante seis noches y observaron que desarrollaron intolerancia a los hidratos de carbono. Este resultado sugiere que el pobre patrón de sueño se relaciona con el desarrollo de intolerancia a los hidratos de carbono y posteriormente de diabetes mellitus, en una población de 53 a 93 años ${ }^{53}$.

Por último, está la asociación entre SAHOS y obesidad. Si bien es cierto que son entidades que con frecuencia se dan juntas, no todos los pacientes con SAHOS son obesos o viceversa. Se ha visto, no obstante, que el índice cintura-cadera, la circunferencia del cuello y la circunferencia de cintura son medidas que predicen mejor el SAHOS que el índice de masa corporal $^{54,55}$. Así mismo se ha observado una correlación fuerte entre la grasa visceral y el $\mathrm{IAH}$, que sugiere que la obesidad central desempeña un papel más importante que la obesidad general en este síndrome, ya que genera mayor carga sobre el sistema respiratorio. Un estudio longitudinal publicado en 2000 reportó que una reducción del 10\% del peso puede llegar a minimizar hasta en $26 \%$ el IAH. Por otra parte, se ha observado que los pacientes que usan CPAP, junto con cambios en sus hábitos alimenticios, muestran una mejor tasa de disminución del peso ${ }^{34}$. Pese a ello, existen casos de obesidad mórbida en los que la única solución para mejorar su perfil metabólico y su trastorno de sueño es la cirugía bariátrica $^{56,57}$.

En 2012, Ruiz et al. publicaron un estudio descriptivo de la prevalencia de síndrome metabólico y obesidad en pacientes con SAHOS en el Hospital Universitario San Ignacio. Se evaluaron 321 pacientes, de los cuales 215 (67\%) fueron diagnosticados con SAHOS y $153(71 \%)$ de estos tenían un índice de masa corporal mayor de 30. De un subgrupo de 93 pacientes, $45(48,4 \%)$ tenían síndrome metabólico y 39 (86,6\%) de ellos SAHOS. A pesar de no haber mostrado significación estadística, probablemente por el bajo tamaño de la muestra, este estudio evidencia la alta coexistencia de SAHOS y síndrome metabólico, al tiempo que resalta la importancia del tratamiento oportuno de estos pacientes con el fin de evitar mayor riesgo cardiovascular ${ }^{57}$.

\section{Situación en Latinoamérica y Colombia}

La asociación entre el SAHOS y las enfermedades cardiovasculares es un tema que ha cobrado auge en los últimos años, si bien en Latinoamérica, y específicamente en Colombia, los estudios reportados hasta la fecha son escasos. La mayoría de la literatura publicada en estos países son revisiones de tema o reportes de caso, y es posible encontrar estudios clínicos en países como México, Brasil, Perú, Chile e incluso Colombia.

Por ejemplo, en 2011 se publicó en la Revista Peruana de Medicina Experimental y Salud Pública un estudio que buscaba evaluar las diferencias entre los pacientes con SAHOS obesos y no obesos. Se concluyó que la severidad del IAH es mayor en pacientes obesos y que estos presentan más comorbilidades; sin embargo reportaron varias limitaciones que sesgaron el estudio, como una población de mujeres insuficiente y que la muestra fue tomada de una clínica de sueño y no de una población general ${ }^{58}$.

En cuanto a la literatura colombiana, en 2008 se realizó en Cali un estudio que mostró la relación entre SAHOS e hipertensión arterial, resultados que concuerdan con las publicaciones internacionales. Sin embargo, este sólo evaluó la asociación y no el SAHOS como un factor de riesgo independiente para desarrollar hipertensión arterial ${ }^{59}$.

\section{Conclusiones}

En los últimos años se ha reconocido al SAHOS como un problema de salud pública mundial. Dadas las circunstancias, así como el alto grado de morbimortalidad que se deriva de las enfermedades cardiovasculares, es imperativo conocer el impacto cardiometabólico de este síndrome para así diagnosticarlo y tratarlo de manera oportuna, pero aún hacen falta estudios clínicos aleatorizados que demuestren 
el impacto del tratamiento sobre los desenlaces cardiovasculares de los pacientes.

Es evidente que tanto en Colombia como en Latinoamérica en general, se requieren estudios prospectivos de cohorte que marquen la pauta para la población del continente y que muestren posibles diferencias respecto a la comunidad internacional.

\section{Conflicto de intereses}

Los autores declaran no tener ningún conflicto de intereses.

\section{Bibliografía}

1. Ryan S, Taylor CT, McNicholas WT. Selective activation of inflammatory pathways by intermittent hypoxia in obstructive sleep apnea syndrome. Circulation. 2005;112(17):2660-7.

2. Quan SF, Howard BV, Iber C, Kiley JP, Nieto FJ, O'Connor GT, et al. The Sleep Heart Health Study: design, rationale, and methods. Sleep. 1997;20(12):1077-85.

3. Peker Y, Hedner J, Norum J, Kraiczi H, Carlson J. Increased incidence of cardiovascular disease in middle-aged men with obstructive sleep apnea: a 7-year follow-up. Am J Respir Crit Care Med. 2002;166(2):159-65.

4. Yeboah J, Redline S, Johnson C, Tracy R, Ouyang P, Blumenthal $\mathrm{RS}$, et al. Association between sleep apnea, snoring, incident cardiovascular events and all-cause mortality in an adult population: MESA. Atherosclerosis. 2011;219(2):963-8.

5. Badran M, Ayas N, Laher I. Cardiovascular complications of sleep apnea: role of oxidative stress. Oxid Med Cell Longev. 2014;2014:985258.

6. Baguet JP, Barone-Rochette G, Tamisier R, Levy P, Pépin JL. Mechanisms of cardiac dysfunction in obstructive sleep apnea. Nat Rev Cardiol. 2012;9:679-88.

7. Vijayan VK. Morbidities associated with obstructive sleep apnea. Expert Rev Respir Med. 2012;6(5):557-66.

8. Selim B, Won C, Yaggi HK. Cardiovascular consequences of sleep apnea. Clin Chest Med. 2010;31(2):203-20.

9. Río Vázquez V, Calderón JA. Mecanismos fisiopatológicos de las alteraciones cardiovasculares en el síndrome de apnea obstructiva del sueño. Rev Cuba Investig Biomédicas. 2009;28(1):1-8.

10. Bagai K. Obstructive sleep apnea, stroke, and cardiovascular diseases. Neurologist. 2010;16(6):329-39.

11. Thomas JJ. Obstructive sleep apnea and cardiovascular complications: perception versus knowledge. Clin Exp Pharmacol Physiol. 2012;39(12):995-1003.

12. Lev́y P, Ryan S, Oldenburg O, Parati G. Sleep apnoea and the heart. Eur Respir Rev. 2013;22:333-52.

13. Pedrosa RP, Krieger EM, Lorenzi-Filho G, Drager LF. Recent advances of the impact of obstructive sleep apnea on systemic hypertension. Arq Bras Cardiol. 2011;97(2):40-7.

14. Torre-Bouscoulet L, Meza-Vargas MS. Riesgo cardiovascular en adultos con síndrome de apnea obstructiva del sueño. A 25 años de los primeros estudios de asociación. Gac Med Mex. 2008;144(4):323-32.

15. Haas DC, Foster GL, Nieto FJ, Redline S, Resnick HE, Robbins JA, et al. Age-dependent associations between sleep-disordered breathing and hypertension: importance of discriminating between systolic/diastolic hypertension and isolated systolic hypertension in the Sleep Heart Health Study. Circulation. 2005;111(5):614-21.

16. Gonçalves SC, Martinez D, Gus M, de Abreu-Silva EO, Bertoluci C, Dutra I, et al. Obstructive sleep apnea and resistant hypertension: a case-control study. Chest. 2007;132(6): 1858-62.
17. Haentjens P, Van Meerhaeghe A, Moscariello A, de Weerdt S, Poppe K, Dupont A, et al. The impact of continuous positive airway pressure on blood pressure in patients with obstructive sleep apnea syndrome. Arch Intern Med. 2007;167: 757-65.

18. Mooe T, Franklin KA, Wiklund U, Rabben T, Holmström K. Sleepdisordered breathing and myocardial ischemia in patients with coronary artery disease. Chest. 2000;117(6):1597-602.

19. Aboyans V, Cassat C, Lacroix P, Tapie P, Tabaraud F, Pesteil F, et al. Is the morning peak of acute myocardial infarction's onset due to sleep-related breathing disorders? A prospective study. Cardiology. 2000;94(3):188-92.

20. Shah NA, Yaggi HK, Concato J, Mohsenin V. Obstructive sleep apnea as a risk factor for coronary events or cardiovascular death. Sleep Breath. 2010;14(2):131-6.

21. Loke YK, Brown JWL, Kwok CS, Niruban A, Myint PK. Association of obstructive sleep apnea with risk of serious cardiovascular events: a systematic review and meta-analysis. Circ Cardiovasc Qual Outcomes. 2012;5(5):720-8.

22. Marin JM, Carrizo SJ, Vicente E. Long-term cardiovascular outcomes in men with obstructive sleep apnoea-hypopnoea with or without treatment with continuous positive airway pressure: an observational study. Lancet. 2005;365(9464):1046-53.

23. Drager LF, Polotsky VY, Lorenzi-Filho G. Obstructive sleep apnea: an emerging risk factor for atherosclerosis. Chest. 2011;140(2):534-42.

24. Drager LF, Bortolotto LA, Lorenzi MC, Figueiredo AC, Krieger EM, Lorenzi-Filho G. Early signs of atherosclerosis in obstructive sleep apnea. Am J Respir Crit Care Med. 2005;172(5):613-8.

25. Nachtmann A, Stang A, Wang YM, Wondzinski E. Association of obstructive sleep apnea and stenotic artery disease in ischemic stroke patients. Atherosclerosis. 2003;169(2):301-7.

26. Drager LF, Bortolotto LA, Figueiredo AC, Krieger EM, Lorenzi GF. Effects of continuous positive airway pressure on early signs of atherosclerosis in obstructive sleep apnea. Am J Respir Crit Care Med. 2007;176(7):706-12.

27. Bayram NA, Ciftci B, Keles T, Durmaz T, Turhan S, Bozkurt E, et al. Endothelial function in normotensive men with obstructive sleep apnea before and 6 months after CPAP treatment. Sleep. 2009;32(10):1257-63.

28. Palomaki H, Partinen M, Juvela S, Kaste M. Snoring as a risk factor for sleep-related brain infarction. Stroke. 1989;20(10):1311-5.

29. Ramar K, Guilleminault C. Cardiovascular complications of obstructive sleep apnea. Expert Rev Respir Med. 2008;2(1):63-74.

30. Arzt M, Young T, Finn L, Skatrud JB, Bradley TD. Association of sleep-disordered breathing and the occurrence of stroke. Am J Respir Crit Care Med. 2005;172(11):1447-51.

31. Munoz R, Duran-Cantolla J, Martínez-Vila E, Gallego J, Rubio R, Aizpuru F, et al. Severe sleep apnea and risk of ischemic stroke in the elderly. Stroke. 2006;37(9):2317-21.

32. Yaggi HK, Concato J, Kernan WN, Lichtman JH, Brass LM, Mohsenin V. Obstructive sleep apnea as a risk factor for stroke and death. N Eng J Med. 2005;353(19):2034-41.

33. MacDonald M, Fang J, Pittman SD, White DP. The current prevalence of sleep disordered breathing in congestive heart failure patients treated with beta-blockers. J Clin Sleep Med. 2008;4(1):38-42.

34. Yumino D, Wang H, Floras JS, Newton GE, Mak S, Ruttanaumpawan $P$, et al. Prevalence and physiological predictors of sleep apnea in patients with heart failure and systolic dysfunction. $J$ Card Fail. 2009;15(4):279-85.

35. Shahar E, Whitney CW, Redline S, Lee ET, Newman AB, Nieto FJ, et al. Sleep-disordered breathing and cardiovascular disease: cross-sectional results of the Sleep Heart Health Study. Am J Respir Crit Care Med. 2001;163(1):19-25.

36. O'Connor GT, Caffo B, Newman AB, Quan SF, Rapoport DM, Redline $\mathrm{S}$, et al. Prospective study of sleep-disordered breathing 
and hypertension: the Sleep Heart Health Study. Am J Respir Crit Care Med. 2009;179(12):1159-64.

37. Dursunoğlu N, Dursunoğlu D, Kiliç M. Impact of obstructive sleep apnea on right ventricular global function: sleep apnea and myocardial performance index. Respiration. 2005;72(3): 278-84.

38. Dursunoglu D, Dursunoglu N, Evrengül H, Ozkurt S, Kuru O, Kiliç $M$, et al. Impact of obstructive sleep apnoea on left ventricular mass and global function. Eur Respir J. 2005;26(2):283-8.

39. Usui K, Bradley TD, Spaak J, Ryan CM, Kubo T, Kaneko $Y$, et al. Inhibition of awake sympathetic nerve activity of heart failure patients with obstructive sleep apnea by nocturnal continuous positive airway pressure. J Am Coll Cardiol. 2005;45(12):2008-11.

40. Bazzano LA, Khan Z, Reynolds K, He J. Effect of nocturnal nasal continuous positive airway pressure on blood pressure in obstructive sleep apnea. Hypertension. 2007;50(2):417-23.

41. Blanco Pérez JJ, Zamarrón Sanz C, Almazán Ortega R, García García M, López Castro J. Síndrome de apnea del sueño en la insuficiencia cardiaca: Efecto de la presión positiva continua en la vía aérea. An Med Intern. 2008;25(1):15-9.

42. Guilleminault C, Connolly SJ, Winkle RA. Cardiac arrhythmia and conduction disturbances during sleep in 400 patients with sleep apnea syndrome. Am J Cardiol. 1983;52(5):490-4.

43. Goyal SK, Sharma A. Atrial fibrillation in obstructive sleep apnea. World J Cardiol. 2013;5(6):157-63.

44. Filgueiras-Rama D, Arias MA, Iniesta A, Armada E, Merino JL, Peinado R, et al. Atrial arrhythmias in obstructive sleep apnea: underlying mechanisms and implications in the clinical setting. Pulm Med. 2013;2013:426758.

45. Baranchuk A. Sleep apnea, cardiac arrhythmias, and conduction disorders. J Electrocardiol. 2012;45(5):508-12.

46. Kanagala R, Murali NS, Friedman PA, Ammash NM, Gersh BJ, Ballman KV, et al. Obstructive sleep apnea and the recurrence of atrial fibrillation. Circulation. 2003;107(20):2589-94.

47. Naruse $Y$, Tada $H$, Satoh $M$, Yanagihara $M$, Tsuneoka $H$, Hirata $Y$, et al. Concomitant obstructive sleep apnea increases the recurrence of atrial fibrillation following radiofrequency catheter ablation of atrial fibrillation: clinical impact of continuous positive airway pressure therapy. Heart Rhythm. 2013;10(3):331-7.

48. Abe H, Takahashi M, Yaegashi H, Eda S, Tsunemoto H, Kamikozawa $M$, et al. Efficacy of continuous positive airway pressure on arrhythmias in obstructive sleep apnea patients. Heart Vessels. 2010;25(1):63-9.

49. Newman AB, Nieto FJ, Guidry U, Lind BK, Redline S, Shahar $E$, et al. Relation of sleep-disordered breathing to cardiovascular disease risk factors: The Sleep Heart Health Study. Am J Epidemiol. 2001;154(1):50-9.

50. Yaggi HK, Araujo AB, McKinlay JB. Sleep duration as a risk factor for the development of type 2 diabetes. Diabetes Care. 2006;29(3):657-61.

51. Sulit L, Storfer-Isser A, Kirchner HL. Differences in polysomnography predictors for hypertension and impaired glucose tolerance. Sleep. 2006;29(6):777-83.

52. Ip MSM, Lam B, Ng MMT, Lam WK, Tsang KWT, Lam KSL. Obstructive sleep apnea is independently associated with insulin resistance. Am J Respir Crit Care Med. 2002;165: 670-6.

53. Gottlieb DJ, Punjabi NM, Newman AB, Resnick HE, Redline $\mathrm{S}$, Baldwin CM, et al. Association of sleep time with diabetes mellitus and impaired glucose tolerance. Arch Intern Med. 2005; 165(8):863-7.

54. Schwartz AR, Patil SP, Laffan AM, Polotsky V, Schneider H. Obesity and obstructive sleep apnea: pathogenic mechanism and therapeutic approaches. Proc Am Thorac Soc. 2008;5(2):185-92.

55. De Sousa AG, Cercato C, Mancini MCHA. Obesity and obstructive sleep apnea-hypopnea syndrome. Obes Rev. 2008;9(4):340-54.

56. Fritscher LG, Mottin CC, Canani S. Obesity and obstructive sleep apnea-hypopnea syndrome: the impact of bariatric surgery. Obes Surg. 2007;17(1):95-9.

57. Ruiz AJ, Hidalgo P, Amado S, Medina L. Prevalencia de síndrome metabólico y obesidad en pacientes con síndrome de apnea hipopnea del sueño (SAHOS) en el Hospital Universitario San Ignacio. Rev Colomb Neumol. 2012;24:18-23.

58. De Castro JR, Rosales-Mayor E. Diferencias clínicas y polisomnográficas entre obesos y no obesos con síndrome de apneas-hipopneas del sueño. Rev Peru Med Exp Salud Publica. 2011;28(4):595-601.

59. Gonzalez LM, Castaño JJ, Herrera V, Jimenez AM, Lentijo P, Sierra A. Relación entre hipertensión arterial sistémica y síndrome de apnea-hipopnea obstructiva del sueño y sus factores de riesgo asociados, en población hipertensa de un centro médico. Arch Med. 2008;8(2):89-97. 